\title{
An Analysis of Cost Allocation and Social Benefits in Reality Shows
}

\author{
Keren Zou \\ Northeast Yucai School, Shenyang 110179, China. \\ Kerenzou2013@sina.com
}

\begin{abstract}
In this paper, cost, revenue, and externalities of reality shows are the mainly considered topics. Based on Cobb Douglas Production Function, the production cost in a reality show can be categorized into two types: fixed cost and variable cost, thus analyzing the revenue and the externalities of the shows. Utilizing the data from a random sample of 10 types of reality shows throughout the nation, 310 volumes in total, as well as Ordinary Least Square technique (OLS), multiple linear regression to analyze, a conclusion can be reached that for draft shows, one available method to increase the program revenue and its positive externality is to aggregate the variable costs such as appearance fee. On the other hand, for the programs of other types, invest more on the fixed cost can attain the same impact.
\end{abstract}

Keywords: Reality show, cost- benefit analysis, externality.

\section{Introduction}

Reality show is a kind of television programs in the form of gaming. It combines the artificiality of the rules, the reality of the participants, and the interactivity of the audience. Originated from Europe and America, reality show was first introduced into China around the 1990s and experienced a progress from imitation to localization. Currently in a stage of zooming, reality shows have developed into tens of types, hundreds of programs, and are broadcast through a wide range of medium like internet, television and so forth. With its rapid growth, a series of commonweal, cultural and value-oriented problems has been unveiled. Scholars in sociology, communication science analyze the reasons for these phenomena and propose their advice. On the foundation of the previous studies, this paper evaluates the economic effect from the cost and revenue aspect, judges the commonweal from the externalities, and advises on the cost allocation for various reality shows.

\section{Literature References}

Vass scholars have made thorough research on promoting the development of reality shows. In this paper, domestic studies can be classified by the views of research.

To begin with, one possible way to enhance the quality of reality shows is to find the solutions towards the drawbacks of a reality show. Representative papers include Du Yue, Lu Ming Tao's articles. [1]

Du Yue (2016) in her paper "Looking at the audience's utility and satisfaction from the star reality show--take 'Running Man' as an example" analyzes the reasons for the popularity of reality shows and their development. Through case analysis, the article attributes the reason for the popularity of the shows to two types: transformation of consumption for stars, and programs satisfying the audience's personal and social aspects of the psychological demand. Finally the article summarizes the reality show's sustainable development strategy: innovate program content, and avoid heavy commercialization. [2] Lu Ming Tao (2016) in his article "A trend analysis of reality shows in the background of Regulations on Reality Shows ", states that the regulation can raise the public participation of reality TV show, enhance culture implanting, enrich plot settings and encourage the original shows; meanwhile, the Internet will promote the development of online variety shows, reform the thinking of traditional program production, and strengthen the interactivity in the programs.

Huang Shanghai in his article "reality TV research overview"[3], through literature analysis, introduces the definition of reality, and claims that content and the moral bottom line should be stressed during localization of reality shows. Moreover, Huang demonstrates the problems of 
domestic reality show at initial stage, mainly due to lack of experience. The solution is to democratize the content, be close to the public, pursue the truth, adhere to the reality, be innovative, and maintain the characteristics. Yin Hong et al. [4] decompose reality show into seven basic elements, including character element, dynamic element, structural element, link element, situation element, detail element and infection element through case analysis in their article "analysis of program elements of reality TV", and carry out detailed analysis one by one in combination with specific programs.

Secondly, distinguished scholars such as Xiang Ixia analyze the audience satisfaction and the influence that the shows exert, which reflects the social attribute of the reality shows. They also propose suggestions to advocate social responsibility. Xiang Ixia in her article "the secrets of reality shows' [5] long life span--the application of the theory of "use and satisfaction" and "Raza Mansfield two-stage transmission theory" shows the audience's psychological demand, and explains the development trend of reality shows and TV production coping strategies, mainly through meeting the audience's psychological needs, improving public participation, and innovating, developing the industrial chain.

Li Nixie you're in her article "using player configuration to show the value orientation of jobhunting reality shows--take 'Only You' in Tianjin TV for example" demonstrates the faults of the play configuration[6]: mainly the exaggeration, hype, and publicity. The author thinks that the program should improve the quality of recruitment, reduce the elements of show making, strengthen the sense of social responsibility so as to establish and enhance the correct orientation. [7]Hai Wei (2011) in his article "the cultural consciousness of the reality TV entertainment era--take the jobhunting reality show "You Are Hired" as an example" the author expounds the cultural dilemma of the development of reality TV entertainment era, criticizes its consumerism, hedonism as the core of the negative impact of culture. However, as the case analysis, "You Are Hired" conforms to the real interpretation of multivariate workplace issues, entertainment and guide of symbiotic win-win situation, the entertainment culture of humanity to ascend. For the future development trend of the reality show, the author makes a revelation: autonomy of cultural choice, culture and the direction of the guide, the importance of cultural literacy.

NieYanYan in her article "the job-hunting reality shows illustrate the return of the social attribute-take "Only You" for example"[8], claims the defects in the current situation of the development of reality shows, as well as applying the case analysis according to the current reality TV to show the return of the social attribute. Only you has found a successful balance between entertainment and sociality, mainly reflected in its response to social heated issues and the host's orientation and guidance, without utilitarian; the program also shows humanistic care but no sensationalism, focuses on the job-hunting and audience experience.

At last, using the experience of foreign reality shows can solve the problems encountered in the development of domestic reality shows. Representative scholars include Pan Xiao Jun. [9] Pan Xiao Jun (2010) in his article "Analysis of American orientation value and its impact on Chinese younger generation orientation values from the view of reality shows", explains the reflection of American orientation values in reality shows, and the influence on the younger generation in China, including opposition and conflict, the unification and convergence. First of all, Pan elaborates the relationship between TV programs and orientation values. Specifically, there are media's shaping of public opinion and the influence of society on media. Secondly, through the comparison between Chinese and American reality shows, the article explains that American reality shows embody the American dream and the pursuit of democracy, freedom and equality. However, the practical value and spiritual value orientation in Chinese reality shows are not clear, which deviates from Chinese traditional and social orientation values. As a result, the opposition, contradiction and conflict of the orientation values of the young generation in China have been triggered. Finally, the author puts forward two solutions: the orientation values reflected in reality shows should seek common ground while reserving differences and persisting in innovation. [10] Ken Nailing (2007) in his article "the development trend of European and American reality TV show and enlightenment", analyzes the development trend of European and American reality TV show, as well as explaining its enlightenment to China's reality. To be more specific, European and American reality shows focus 
on reality but not bragging, reflecting the positive theme, containing a variety of themes: comprehensive performance, languages, reality, and other elements, culture tends to local, and keeping in line with the mainstream social orientation values.

\section{Models and Data}

\subsection{Model}

Based on the Solow model, this paper analyzes the influence of input factors on production participants from the perspective of manufacturers and consumers. For reality shows, it is essential to compare TV channels as producers and audience as consumers. The analysis of reality show producers such as TV stations and network platforms mainly focuses on the analysis of their costs and benefits. The details are shown in formula (3). According to Cobb Douglas Production Function, this paper divides the cost of reality shows into two types, including fixed cost and variable cost. The effects on the income of programs are analyzed respectively, so as to measure the profit maximization behaviors of program producers like TV stations and network platforms. For the analysis of audience effect, this paper mainly deploys two indicators -- audience rating and clicks volume to measure. Audience rating refers to the percentage of the total number of TV viewers (or households) who watch a certain TV channel (or channel where a reality show is located) within a certain period of time. Click volume refers to the number of times a reality show has been clicked on in a certain period of time on the Internet. The advantage of using audience rating to measure audience effect is that the passive communication mode of traditional media can cover audiences of all ages and make the data universally representative. The advantage of using to measure the audience effect is that the active communication mode of emerging media can effectively reflect the popularity of reality shows and the positive effect brought to the audience.

Solow model:

$$
\mathrm{Y}=\mathrm{AF}(\mathrm{K}, \mathrm{L})
$$

Cobb Douglas Production Function:

$$
\begin{aligned}
& \log ^{Y}=\log ^{A}+\alpha \log ^{K}+\beta \log ^{L} \\
& \mathrm{InC}=\mathrm{A}(F C)^{\alpha}(V C)^{\beta} \\
& \mathrm{ADr}=\mathrm{A}(F C)^{\alpha}(V C)^{\beta} \\
& \mathrm{CKq}=\mathrm{A}(F C)^{\alpha}(V C)^{\beta}
\end{aligned}
$$

\subsection{Data}

The data used in this paper come from the following three channels: first, the data of TV program ratings come from the official data of The State Administration of Radio, Film and Television. Secondly, the data of the cost and benefit of TV programs come from the work reports issued by local TV stations. Finally, the reason why network platform programs use a variety of ways to collect data of the reality TV show is that there is no determined official channel at present stage in our country. Whereas The State Administration of radio and local TV stations can provide data represented by television end of traditional media, but the rise of new media such as network, allows more viewers through the network video platform to watch programs, which makes the official figures represented by the state administration of radio channel exists omission. On the basis of considering data authority and comprehensiveness, this paper uses the data collected from the three channels so as to better meet the demand of the research model for data. 


\section{Summary}

\subsection{Analysis of Regression Results}

In this paper, the least squares method (OLS) is used to carry out regression analysis for equations (3), (4) and (5) respectively. The regression results of equation (3) as shown in table 1 (see table 1), the return of the dependent variable to reality TV show income (income), regression independent variables including programming (FC) fixed cost and variable cost (VC); Regression results of the first column represents 10 types in the database, a total of 310 the return of the reality TV show as a result, the second column represents the entertainment shows the regression results, the third column represents the performing talent shows the regression results, the fourth column represents the regression results of indoor reality show, the fifth column represents the regression results of jobhunting shows, the sixth column represents the regression results of public welfare type of reality TV show, the seventh column represents the wild shows the regression results, the eighth column represents the regression results of travelling shows, the ninth column represent the regression results of law-related reality show, the first ten columns represent the regression results of educational shows, the eleventh column represent the regression results of dating shows. From the overall regression results, the fixed cost has a significant positive impact on the program income, with an impact coefficient of 1.37. Variable costs also had a significantly positive impact on program revenue, with an impact coefficient of 12.34. As far as each kind of reality show is concerned, fixed cost investment has a significant positive impact on all programs, and it has the largest impact on reality shows performing talent shows, which is 16.5 . Variable costs have different effects on different types of reality shows. Regression results show that variable costs have the greatest impact on entertainment and talent shows.

Table 1 . The regression results of audience rate (Ad) in reality shows (OLS)

\begin{tabular}{ccccccc}
\hline \multirow{2}{*}{ Variables } & $(1)$ & $(2)$ & $(3)$ & $(4)$ & $(5)$ & $(6)$ \\
& inc & inc & inc & inc & inc & inc \\
\hline \multirow{2}{*}{ fc } & $1372^{* * *}$ & $14.53 * * *$ & $16.50^{* * *}$ & $5.140^{* * *}$ & $4.707 * * *$ & $5.383^{* * * *}$ \\
& $(0.129)$ & $(1.330)$ & $(1.543)$ & $(0.542)$ & $(0.458)$ & $(0.498)$ \\
vc & $1234^{* * *}$ & $3.748^{* * *}$ & $4.024 * * *$ & $-5.521^{* *}$ & $-4.891 * *$ & $-5.358^{* *}$ \\
& $(0.428)$ & $(1.144)$ & $(1.243)$ & $(2.593)$ & $(2.194)$ & $(2.204)$ \\
Constant & -275.2 & $-1,567$ & $-1,760$ & -707.3 & -691.3 & -807.7 \\
& $(430.7)$ & $(1,210)$ & $(1,143)$ & $(953.6)$ & $(1,048)$ & $(773.3)$ \\
Observations & 310 & 31 & 31 & 31 & 31 & 31 \\
R-squared & 0.856 & 0.958 & 0.956 & 0.954 & 0.955 & 0.959 \\
\hline \multirow{5}{*}{} & $(7)$ & $(8)$ & $(9)$ & $(10)$ & $(11)$ & \\
\hline & inc & inc & inc & inc & inc & \\
& $6.143 * * *$ & $5.438^{* * *}$ & $6.499 * * *$ & $6.329 * * *$ & $5241 * * *$ & \\
& $(0.690)$ & $(0.498)$ & $(0.917)$ & $(1.006)$ & $(1.048)$ & \\
& $-8.007 * *$ & $-6.080^{* *}$ & $-8.564 *$ & -7.542 & -2.197 & \\
& $(3.371)$ & $(2.312)$ & $(4.483)$ & $(4.858)$ & $(5.172)$ & \\
& -656.4 & -737.9 & -657.7 & $-740.5 *$ & -588.9 & \\
& $(583.0)$ & $(621.6)$ & $(513.2)$ & $(405.8)$ & $(352.3)$ & \\
& 31 & 31 & 31 & 31 & 31 & \\
& 0.959 & 0.963 & 0.956 & 0.959 & 0.957 & \\
\hline
\end{tabular}

Standard errors in parentheses $* * * \mathrm{p}<0.01, * * \mathrm{p}<0.05, * \mathrm{p}<0.1$.

There is a negative influence on other kinds of reality shows, among which the influence on the dating shows is negative but not significant. The regression results of table 2 (see attached table 2) 
show the relationship between the ratings of reality shows and their costs, as shown in equation (4). The first column represents the regression results of a total of 310 reality shows in 10 categories in the data. From the perspective of regression coefficient, the fixed cost (FC) has a significant positive influence on the program rating, and the influence coefficient is $1.47 \mathrm{e}-06$.Variable cost (VC) also has a significant positive impact on program ratings, with an impact coefficient of 4.51e-06. Specific to each type of reality TV show, fixed costs increased ratings for all programs have significant positive effects, including performance for draft class of reality TV show has the greatest effect in the ratings, and influence coefficient is $1.33 \mathrm{e}-05$. Variable costs for the program's ratings and the influence of the fixed cost is different, the acting talent shows, although there is a positive influence but not, significantly, on the other nine types of reality TV show has a negative effect, but the negative effect is only significant effects of two types of reality TV show, workplace reality show and dating reality shows. Show the relationship between click volume and cost of reality TV programs, as shown in equation (5). Ten classes, a total of 310 in the first column represents the data phase of the return of the reality TV show as a result, from the point of regression coefficient, fixed costs (FC) for program hits have significant positive influence, influence coefficient is 1.986; Variable cost (VC) also has a significant positive impact on program clicks, with an impact coefficient of 16.64. Specific to each type of reality TV show, fixed cost increase for all show hits has significant positive influence; among them to show the draft show the biggest influence on, the influence coefficient of 40.84 . Variable costs for the program click on the quantity and the influence of fixed cost is different, the acting talent shows and travel type of reality TV show has a positive effect, the former (9.858) (0.132) is more significant than the latter, while the other eight kind of reality TV show has the negative influence of big or small, reality show negative effect which the two most significant - education reality show and dating reality shows.

Table 2. The regression results of click quantity (Cake) in reality shows

\begin{tabular}{|c|c|c|c|c|c|c|}
\hline Variables & $\begin{array}{c}(1) \\
\text { ADrate }\end{array}$ & $\begin{array}{c}(2) \\
\text { ADrate }\end{array}$ & $\begin{array}{l}\text { (3) } \\
\text { ADrate }\end{array}$ & $\begin{array}{c}\text { (4) } \\
\text { ADrate }\end{array}$ & $\begin{array}{c}\text { (5) } \\
\text { ADrate }\end{array}$ & $\begin{array}{c}(6) \\
\text { ADrate }\end{array}$ \\
\hline \multirow[t]{2}{*}{ fc } & $1.47 \mathrm{e}-06^{* * *}$ & $8.70 \mathrm{e}-06^{* * *}$ & $1.33 \mathrm{e}-05^{* * *}$ & $4.06 \mathrm{e}-06^{* * *}$ & $1.95 \mathrm{e}-06^{* * *}$ & $4.41 \mathrm{e}-06^{* * *}$ \\
\hline & $(2.00 \mathrm{e}-07)$ & $(9.96 \mathrm{e}-07)$ & $(3.20 \mathrm{e}-06)$ & $(1.11 \mathrm{e}-06)$ & $(3.78 \mathrm{e}-07)$ & $(1.17 \mathrm{e}-06)$ \\
\hline $\mathrm{vc}$ & $\begin{array}{c}4.51 \mathrm{e}-06 * * * \\
(6.68 \mathrm{e}-07)\end{array}$ & $\begin{array}{r}-1.50 \mathrm{e}-06^{*} \\
(8.56 \mathrm{e}-07)\end{array}$ & $\begin{array}{r}1.88 \mathrm{e}-06 \\
(2.57 \mathrm{e}-06)\end{array}$ & $\begin{array}{l}-4.41 \mathrm{e}-06 \\
(5.31 \mathrm{e}-06)\end{array}$ & $\begin{array}{c}-4.29 \mathrm{e}-06 * * \\
(1.81 \mathrm{e}-06)\end{array}$ & $\begin{array}{r}-3.38 \mathrm{e}-06 \\
(5.18 \mathrm{e}-06)\end{array}$ \\
\hline \multirow[t]{2}{*}{ Constant } & $0.00365^{* * *}$ & 0.000913 & $6.33 \mathrm{e}-05$ & 0.000736 & 0.00111 & 0.000520 \\
\hline & $(0.000671)$ & $(0.000906)$ & $(0.00237)$ & $(0.00195)$ & $(0.000866)$ & $(0.00182)$ \\
\hline Observations & 310 & 31 & 31 & 31 & 31 & 31 \\
\hline \multirow[t]{10}{*}{ R-squared } & 0.417 & 0.865 & 0.734 & 0.755 & 0.742 & 0.762 \\
\hline & $\begin{array}{c}(7) \\
\text { ADrate }\end{array}$ & $\begin{array}{c}(8) \\
\text { ADrate }\end{array}$ & $\begin{array}{c}(9) \\
\text { ADrate }\end{array}$ & $\begin{array}{c}\text { (10) } \\
\text { ADrate }\end{array}$ & $\begin{array}{c}\text { (11) } \\
\text { ADrate }\end{array}$ & \\
\hline & $5.46 \mathrm{e}-06 * * *$ & $4.15 \mathrm{e}-06^{* * *}$ & $7.60 \mathrm{e}-06^{* * *}$ & $7.75 \mathrm{e}-06^{* * *}$ & $1.31 \mathrm{e}-05^{* * *}$ & \\
\hline & $(1.53 \mathrm{e}-06)$ & $(1.15 \mathrm{e}-06)$ & $(2.34 \mathrm{e}-06)$ & (2.61e-06) & $(2.99 \mathrm{e}-06)$ & \\
\hline & $-6.31 \mathrm{e}-06$ & $-2.22 \mathrm{e}-06$ & $-1.17 \mathrm{e}-05$ & $-1.01 \mathrm{e}-05$ & $-3.14 \mathrm{e}-05^{* *}$ & \\
\hline & $(7.48 \mathrm{e}-06)$ & $(5.33 \mathrm{e}-06)$ & $(1.14 \mathrm{e}-05)$ & $(1.26 \mathrm{e}-05)$ & $(1.47 \mathrm{e}-05)$ & \\
\hline & 0.000601 & 0.000510 & $2.94 \mathrm{e}-05$ & -0.000141 & 0.000277 & \\
\hline & $(0.00129)$ & $(0.00143)$ & $(0.00131)$ & $(0.00105)$ & $(0.001(8))$ & \\
\hline & 31 & 31 & 31 & 31 & 31 & \\
\hline & 0.802 & 0.790 & 0.801 & 0.829 & 0.852 & \\
\hline
\end{tabular}

Standard errors in parentheses $* * * \mathrm{p}<0.01, * * \mathrm{p}<0.05, * \mathrm{p}<0.1$.

\subsection{Conclusions}

First of all, for reality show producers, the biggest concern is to maximize their income. Through regression analysis result, this paper finds that for common reality shows, the increase of both fixed 
costs and variable costs can effectively increase the income. Compared with the fixed cost, variable cost makes greater difference. Among them, for performing talent shows, increasing variable costs, such as inviting more famous stars to increase the star appearance fee, can effectively increase the program income. For other types of reality shows, such as job-hunting reality shows, increasing variable costs, such as star appearance fees, will have a negative impact on program revenue. At the same time, for education and dating reality shows, the increase in star appearance fees and other expenses has no significant impact on the program's income. Secondly, for the audience rating, one of the representatives of the external effects of the program, the regression results show that both increasing fixed costs and variable costs can effectively improve the audience rating of the program. But the fixed and variable costs for different types of reality TV ratings have different effects, including fixed cost increase for all reality TV ratings have significant positive influence, for the draft class has the greatest effect in the reality show; Variable cost changes in ratings is reality because of the influence of different types and different, for performing draft shows variable cost increases to a certain extent can improve the ratings, for other type of reality TV show, the increase of the variable cost, such as the rise in the cost of star appearance, etc., to show the ratings have no significant negative effects. Finally, for the click volume, another representative of the external effect of the program, regression results show that both increasing fixed cost and variable cost expenditure can effectively increase the click volume of the program. Compared with variable costs, fixed costs have a more significant impact on program clicks. Specific to each kind of reality show, the increased cost of fixed clicks for the program has a positive impact, while the variable cost impact on different kinds of reality TV show is different - for performing draft shows and travelling reality shows, it has a positive impact, while for other kinds of reality television network traffic has a negative impact, although this effect is not obvious.

\section{Suggestions and Prospects}

Combined with the conclusion of the empirical analysis, from two perspectives, program producers and audience in this paper, suggestions are proposed on the basis of both revenue and audience effect, so that program producers and regulators can take the following measures:

First, producers can adjust their fixed and variable cost ratios according to the type of reality shows they produce. Specifically, performances and other entertainment draft reality show can appropriately increase the variable cost, such as inviting increased star big star appearance fees, etc., thus improve the show income; for public welfare reality shows like education in the workplace, increasing fixed cost expenditure can effectively increase program income. Secondly, from the perspective of audience effectiveness, program supervisors can make adjustments and Suggestions on the types and cost composition of reality shows. Specific to different types of reality TV show, from the point of view of the ratings, increase the variable cost for viewing generally no significant effect, suggesting that the star utility has not been effective reflection. In contrast, increasing fixed costs can effectively increase the viewing rate of programs. From the perspective of the audience, the producer of the program should appropriately increase the proportion of fixed costs in the total cost. From the point of view of click volume, click volume can reflect the external effect of reality show more than audience rating. Specific to different types of reality TV show, the increase in the variable costs to draft class performance hits have significant positive influence, it shows that network video platform audience nod to star effect is more; But for other types of reality TV show, variable cost impact on network traffic are not obvious, so the reality TV producers in the acting talent shows, also should allocate a larger proportion in fixed costs.

In this study, there are the following problems: the first is that the data on the reality TV show is in the midst of the vigorous development of stage in our country, the lack of a unified the official statistics caliber, shows the information such as the cost of income is not transparent. On the basis of obtaining more data, subsequent studies can make a deeper analysis on the cost of programs. Secondly, on the model, different types of reality shows have different characteristics in the development 
process. This paper adopts a unified model for analysis. Subsequent studies can refine different types of real it shows and adopt different models to reflect the differences between programs.

\section{References}

[1]. Du Yue, 2014, Looking at the audience's utility and satisfaction from the star reality show--take "Running Man" as an example, Selected Papers of Students, the 12th issue of 2014 [J].

[2]. Lu Ming Tao, 2016, A trend analysis of reality shows in the background of Regulations on Reality Shows, Southeast Communication, the 1st issue of 2016 [J].

[3]. Huang Shanghai, 2010, Research summary of reality TV, Journalism University, 2010, the 3rd issue of $2010[\mathrm{~J}]$.

[4]. Yin Hong, Lu Hong, Ran Rogue, 2005, Analysis of program elements of reality TV, Communication Art and Art Communication, 2005, issue 5 [J].

[5]. Xiang Ixia, 2013, the secrets of reality shows' long life span-- the application of the theory of "use and satisfaction, Young Writer, Film and Television Literature, the 4th issue of 2013 [J].

[6]. Li Nuncio your, Li Qumbu, 2012, Using player configuration to show the value orientation of job-hunting reality shows-- take 'Only You' in Tianjin TV for example, Hubei Social Science, the 7 th issue of $2012[\mathrm{~J}]$.

[7]. Hai Wei, 2011, The cultural consciousness of the reality TV entertainment era--take the jobhunting reality show "You Are Hired" as an example, Journal of Fusing university of Fujian Normal University, the 6th issue of 2011 [J].

[8]. Nia Banyan, 2011, The job-hunting reality shows illustrate the return of the social attribute --take "Only You" for example, The World of Sound Screen, the 9th issue of 2011 [J].

[9]. Pan Xiao Jun, 2010, Analysis of American orientation value and its impact on Chinese younger generation orientation values from the view of reality shows, Typical Research the 11th issue of $2010[\mathrm{~J}]$.

[10]. Ken Nailing, 2007, the development trend of European and American reality TV show and enlightenment, Typical Research, the 7th issue of 2007 [J]. 